\title{
SOME PARTIAL UNIT MEMORY CONVOLUTIONAL CODES
}

\author{
Khaled Abdel-Ghaffar \\ University of California \\ Davis, CA 95616
}

\author{
Robert McEliece \\ California Institute of Technology \\ Pasarlcra, CA 91125
}

Gustave Solomon

10747 Wilshire Blvd.

Los Angeles, CA 90024

\section{Summary}

In general, an $[n, k, d ; m]$ convolutional code over a field $F$ has generator matrix $G(D)=G_{0}+G_{1} D+\cdots+G_{K} D^{K}$, where each $G_{i}$ is a $k \times n$ matrix with entries from $F$. Here $n$ is the branch length, $k$ is the dimension per branch, $m$ is the memory (i.e., the total number of nonzero rows in the matrices $G_{1}, \ldots, G_{K}$ ), and $d$ is the free distance. Thus in this notation an $[n, k, d]$ block code is a $[n, k, d ; 0]$ convolutional code. A partial unit memory (PUM) convolutional code is one for which $K=1$ (hence the term "unit memory") and at least one of the rows of $G_{1}$ is zero (hence the term "partial unit memory.") Indeed, if the first $k-m$ rows of $G_{1}$ are all zero, then the resulting code is a $[n, k, d ; m] \mathrm{PUM}$ code.

In this paper we will give a general construction for partial unit memory convolutional codes. This construction may be used to design efficient finite state codes [2], [3]. Informally, the construction goes like this: Suppose $\mathcal{C}^{*}$ and $\mathcal{C}_{0}$ are two linear block codes of length $n$, with $\mathcal{C}^{*} \subseteq \mathcal{C}_{0}$. Suppose $\mathcal{C}^{*}$ is a $\left[n, k^{*}, d^{*}\right]$ code, and $\mathcal{C}_{0}$ is a $\left[n, k, d_{0}\right]$ code. Then almost always we can combine these two codes to make a noncatastrophic partial unit memory convolutional code with parameters $\left[n, k, d ; k-k^{*}\right]$, where $d \geq \min \left(d^{*}, 2 d_{0}\right)$. Formally, the construction is described in the following theorem.

Theorem 1. Suppose that $\mathcal{C}_{0}$ is an $\left[n, k, d_{0}\right]$ linear block code, and that $\mathcal{C}_{1}$ is an $\left[n, k, d_{1}\right]$ linear block code, and $\mathcal{C}_{0} \neq \mathcal{C}_{1}$. Suppose further that $\mathcal{C}_{0}$ and $\mathcal{C}_{1}$ contain a common subcode $\mathcal{C}^{*}$ which is a $\left[n, k^{*}, d^{*}\right]$ code. Then there exists a noncatastrophic $[n, k, d ; m]$ PUM convolutional code, with $m=k-k^{*}$ and $d \geq \min \left(d^{*}, d_{0}+d_{1}\right)$.

In applications, almost always (but not always) we only need two codes, $\mathcal{C}^{*}$ and $\mathcal{C}_{0}$. This is because as a rule the automorphism group of $\mathcal{C}^{*}$ will contain a permutation $\pi$ that does not fix $\mathcal{C}_{0}$, and we can take $\mathcal{C}_{1}=\mathcal{C}_{0}^{\pi}$ in Theorem 1 . The following Corollary spells this out.

Corollary 1. Suppose that $\mathcal{C}_{0}$ is an $\left[n, k, d_{0}\right]$ linear block code, and that $\mathcal{C}^{*}$ is a $\left[n, k^{*}, d^{*}\right]$ code which is a subcode of $\mathcal{C}_{0}$. If the automorphism group of $\mathcal{C}^{*}$ contains a permutation that does not fix $\mathcal{C}_{0}$, then there exists a $[n, k, d ; m] P U M$ convolutional code, with $m=k-k^{*}$ and $d \geq \min \left(d^{*}, 2 d_{0}\right)$.

Theorem 1 and Corollary 1 permit us to construct a large number of PUM codes, many of which are optimal, in the sense of having the largest possible $d_{\text {free }}$ for the given $n, k$, and $m$. Here are two Examples.

Example 1 . Let $\mathcal{C}^{*}$ be the $[8,1,8]$ binary repetition code, and let $\mathcal{C}_{0}$ be the $[8,4,4]$ extended Hamming code. The automorphism group of $\mathcal{C}^{*}$ is the symmetric group $S_{8}$, which plainly does not fix $\mathcal{C}_{0}$. Thus Corollary 1 implies the existence of a $[8,4,8 ; 3]$ PUM code, which is optimal. This code was previously known (see e.g. [1]), but it is interesting to see how easily our construction finds it. It is also the inner code in the well-known Soviet concatenated "Regatta" system.

Example 2. Let $\mathcal{C}_{0}$ be the binary Golay $[24,12,8]$ code. It is possible to show that there is an isomorphic copy of $\mathcal{C}_{0}$, which we call $\mathcal{C}_{1}$, such that the dimension of the intersection $\mathcal{C}_{0} \cap \mathcal{C}_{1}$ is 9 . This intersection contains both a $[24,5,12]$ code, and a $[24,2,16]$ code. Thus by Theorem 1 we can construct both a $[24,12,12 ; 7]$ PUM code, and a $[24,12,16 ; 10]$ PUM code, which are both optimal.

In the special case that $\mathcal{C}^{*}$ is the $[n, 1, n]$ binary repetition code (as in Example 1), the automorphism group of $\mathcal{C}^{*}$ contains all permutations on $\{1,2, \ldots, n\}$. Then unless $k=1, n-1$, or $n, \mathcal{C}_{0}$ can't be fixed by all such permutations. This leads to the following Corollary to Theorem 1.
Corollary 2. If $\mathcal{C}_{0}$ is a $\left[n, k, d_{0}\right]$ binary block code containing the all-ones vector, and if $k \neq 1, n-1, n$, then there exists a $[n, k, d ; k-1]$ $P U M$ code with $d \geq 2 d_{0}$.

Corollary 2 naturally leads one to ask how large can $d_{0}$ be, given that $\mathcal{C}_{0}$ contains the all-ones vector. We do not have a full answer to this question, but the following modification of the classic Griesmer bound is useful

Thus let $N(k, d)$ denote the minimum length of a binary code with Hamming distance $\geq d$ and dimension $k$ which contains the all-ones vector.

Theorem 2. If $k \geq 2$, then

$$
N(k, d) \geq d+N(k-1,\lceil d / 2\rceil) .
$$

Corollary 3. $N(1, d)=d$, and $N(2, d)=2 d$, and for $k \geq 3$,

$$
N(k, d) \geq d+\lceil d / 2\rceil+\left\lceil d / 2^{2}\right\rceil+\cdots+\left\lceil d / 2^{k-3}\right\rceil+2\left\lceil d / 2^{k-2}\right\rceil .
$$

Theorem 2 proves, for example, that there is no $[7,3,4]$ binary code containing the all-ones vector, although there is a $[7,3,4]$ code. Similarly, there is no $[20,5,9]$ linear code with the all-ones vector, although there is an $[21,5,9]$ such code. This is of interest, since Lauer [1] constructed a $[20,5,18 ; 4]$ PUM code, which therefore cannot be constructed by our methods. However, all of Lauer's other codes, and many others scattered throughout the literature, can be constructed by our methods. Theorem 2 also raises the following question: Give a bound on the minimum distance of a linear block code that contains a known subcode. Except for the special case where the subcode is the repetition code, we know practically nothing about this question.

\section{Acknowledgements}

Abdel-Ghaffar's contribution to this paper was supported by NSF Grant NCR 89-08105 and by an IBM Faculty Development Award. McEliece's contribution was partially supported by a consulting contract with Caltech's Jet Propulsion Laboratory under contract to the National Aeronautics and Space Administration, and also partially supported by AFOSR grant 88-0247 and a grant from Pacific Bell. Solomon's contribution was supported by a consuling contract with Caltech's Jet Propulsion Laboratory under contract to the National Aeronautics and Space Administration.

\section{References}

[1] Lauer, G. S., "Some Optimal Partial-Unit-Memory Codes," IEEE Trans. Inform. Theory vol. IT-25 (March 1979), pp. 240243

[2] Pollara, F., McEliece, R., and Abdel-Ghafar, K., "Finite-State Codes," IEEE Trans. Inform. Theory vol. IT-34 (September 1988), pp. 1083-1088.

[3] Pollara, F., Cheung, K.-M., and McEliece, R. J., "Further Results on Finite-State Codes," TDA Progress Report vol. 42-92 (October-December 1987), pp. 56-62. 